\title{
Article
}

\section{Moving Beyond Traditional Understandings of Evidence-Based Practice: A Total Evidence and Knowledge Approach (TEKA) to Treatment Evaluation and Clinical Decision Making in Speech- Language Pathology}

Roddam, Hazel, McCurtin, Arlene and Murphy, Carol-Anne Available at http://clok.uclan.ac.uk/29106/

Roddam, Hazel ORCID: 0000-0002-0637-1801, McCurtin, Arlene and Murphy, Carol-Anne (2019) Moving Beyond Traditional Understandings of EvidenceBased Practice: A Total Evidence and Knowledge Approach (TEKA) to Treatment Evaluation and Clinical Decision Making in Speech-Language Pathology. Seminars in Speech and Language, 40 (05). pp. 370-393. ISSN 0734-0478

It is advisable to refer to the publisher's version if you intend to cite from the work. http://dx.doi.org/10.1055/s-0039-1694996

For more information about UCLan's research in this area go to http://www.uclan.ac.uk/researchgroups/ and search for < name of research Group>.

For information about Research generally at UCLan please go to http://www.uclan.ac.uk/research/

All outputs in CLoK are protected by Intellectual Property Rights law, including Copyright law. Copyright, IPR and Moral Rights for the works on this site are retained by the individual authors and/or other copyright owners. Terms and conditions for use of this material are defined in the policies page. 
TEKA

Moving beyond traditional understandings of evidence based practice: A total evidence and knowledge approach (TEKA) to treatment evaluation and clinical decision-making in speech-language pathology.

Arlene McCurtin $\mathrm{PhD}^{1,2}$ Carol-Anne Murphy $\mathrm{PhD}^{1,2}$ Hazel Roddam $\mathrm{PhD}^{3}$

${ }^{1}$ School of Allied Health, University of Limerick, Limerick, Ireland

${ }^{2}$ Health Research Institute, University of Limerick, Limerick, Ireland

${ }^{3}$ University of Central Lancashire, Preston, Lancashire, UK.

Corresponding author: Dr Arlene McCurtin, School of Allied Health, Health Sciences Building, University of Limerick, Limerick, Ireland. Arlene.mccurtin@ ul.ie 0035361 234180 
TEKA

Abstract

Evidence-based practice (EBP) is a well-established framework for supporting clinical decision-making in the discipline of speech-language pathology. The benefits of using evidence to inform clinical practice are acknowledged by clinicians and researchers alike. Even so, after over two decades of EBP advocacy, much clinical uncertainty remains and models supporting the evaluation of interventions require review and reconsideration. The EBP model, while promoting positive principles, can be argued to be conceptually flawed because it suffers from a lack of attention to and explicit valuing of other forms of knowledge crucial to the formation of realistic and judiciously informed decisions. We propose that the evaluation of interventions would be better supported by an explicit knowledge management approach reflecting a range of evidence and knowledge. One worked example is presented to demonstrate what using such an approach can produce in terms of intervention information.

Keywords: knowledge, evidence, evidence-based practice, intervention evaluation, clinical decision making, critical thinking

Conflicts of interest: The authors confirm they have no conflicts of interest

\section{Disclosures:}

Financial: No relevant financial relationships exist for any of the authors

Nonfinancial: No relevant nonfinancial relationships exist for any of the authors 
TEKA

\section{Introduction}

Evidence Based Practice

Evidence-based practice (EBP) has been a well-established framework for supporting clinical decision-making in the discipline of speech-language pathology (SLP) as well as other in health professions since the late 1990s. EBP, which originated as evidence based medicine (EBM) in the medical profession, had a number of drivers. These included concerns about a reliance on expert judgement with its inherent variability, as well as the use of ineffective therapies in healthcare. ${ }^{1}$ Government and healthcare insurance agencies started to increasingly demand factual evidence for medical treatments, so that they could have a more objective basis for procuring the most clinically and economically effective healthcare available. Such political and financial drivers pushed forward the EBM agenda in order to assure greater consistency and continuity of best practice and effective healthcare treatments. ${ }^{2}$ The EBM movement, emphasizing that clinical decision-making should focus more explicitly on high quality scientific evidence, rather than on clinical intuition, was closely followed across nursing and the allied health professions. ${ }^{3}$ Professional associations, including those in speech and language pathology, now almost universally actively promote the EBP agenda. It is also linked with regulatory requirements for individuals to undertake continuing education (CE) and for services to be accountable for clinical effectiveness. The impact of EBP has been significant across all healthcare disciplines, and it has been labelled the 'most important contemporary initiative committed to reshaping biomedical reason and practice ${ }^{4}$ (p. 1059).

What is evidence based practice exactly?

The most widely accepted original definition of EBM is that first published by David Sackett and colleagues ${ }^{5}$ which identified three distinctive components or 'pillars' of EBM: 
TEKA

best quality research evidence, experienced clinical judgement and factors relating to the individual patient. Dollaghan ${ }^{6}$ perhaps provides the clearest definition of EBP: 'The conscientious, explicit and judicious integration of 1) best available external evidence from systematic research, with 2) best available evidence internal to clinical practice, and 3) best available evidence concerning the preferences of a fully informed patient' (p. 2). All three pillars were intended to be considered in balance and to be given equal weight - most often represented visually as three overlapping circles, or as the three points of a triangle; as seen in ASHA's model. $^{7}$

\section{The primacy of efficacy evidence}

Despite the good intentions of EBP, as $\mathrm{McCabe}^{8}$ has recently argued "we seem to be no closer to closing the gap between research evidence and practice" (p. 300). There are a number of possible explanations to account for this. They include conceptual issues with the EBP model itself ${ }^{9}$ and an unintended and predominant focus on efficacy evidence over and above the other two pillars, with a perceived primacy for the experimental research paradigm. Positivist research designs alone however, are insufficient to fully reflect the effectiveness, impact and patient experience of complex interventions for highly heterogeneous populations. Dollaghan ${ }^{6}$ contends that this 'emphasis on scientific evidence has overshadowed the other two components' (p. 2) while McCurtin ${ }^{10}$ argues that EBP places a hierarchical value on certain types of evidence (e.g., RCTs) resulting in the model suffering from a lack of attention to, and development of, practice and patient evidence. Knowledge and evidence generated by clinicians and patients is crucial to the formation of realistic and judiciously informed decisions. The inherent assumption in EBP that valid knowledge emanates primarily from efficacy evidence can be said to be incompatible with the realities of clinical practice. This may, in part, explain difficulties with evidence implementation, 
TEKA

impacting our potential to make reasonably informed decisions. Further, while some areas of SLP practice such as phonological interventions now have a relatively strong empirical evidence-base $\mathrm{e}^{11,12}$ compared with some other areas of speech and language pathology practice, there is a low likelihood that the discipline's evidence base will ever be complete and that efficacy evidence will ever be sufficient to support treatment evaluations. ${ }^{1,13}$

\section{The complexities of intervening}

We contend that EBP insufficiently acknowledges the complexities of the treatment process and the multiplicity of factors that can impact treatment outcomes. These include dynamics such as the therapeutic alliance and the placebo effect. ${ }^{14,15}$ Furthermore, as Plesk and Greenhalgh ${ }^{16}$ point out, clinical uncertainty and ambiguity are normal components of intervening. The context within which we provide intervention is also increasingly acknowledged as pivotal ${ }^{17 .}$ This includes organisational and environmental contexts and drivers such as funding, access to services, and the training and supports provided to practitioners. ${ }^{18-20}$ Even with empirically supported treatments, Greenhalgh and Maskrey 9 maintain that statistically significant benefits may be marginal in clinical practice and the inflexible protocols attached to some interventions may not be patient-centred. Thus, even the best efficacy evidence may be inapplicable to, or inappropriate for an individual patient or context.

Given all this, it is unlikely that EBP alone can ever be entirely effective in answering all clinical dilemmas. As Berg \& Slaattelid ${ }^{21}$ assert, using a research-only lens to evaluate interventions is "a reductive conceptualisation of intervention" (p. 1). In essence, simplifying treatment to its efficacy base fails to reflect the complexities of the treatment process, limiting our understanding of what contributes to therapy outcomes. Thus, the process of 
TEKA

intervening is the sum of a number of complex interacting components, and treatment outcomes cannot be solely explained by, or attributed to, the treatment or technique applied.

\section{Promoting a broad-based epistemological approach to evaluating interventions}

We argue that the evaluation of interventions requires a broad-based epistemological approach, reflecting multiple forms of knowledge and expanding on the range and depth of information conventionally used in EBP. The strength, legitimacy and validity of treatment evaluations and decisions can be bolstered when an explicit knowledge gathering approach is employed. Further, not engaging with a range of evidence and knowledge can result in much valuable information being lost to the treatment evaluation and decision-making processes and may impact treatment outcomes.

Knowledge or evidence synthesis endeavours to summarize all relevant studies on a specific question. It can contribute to the understanding of discrepancies in the various evidence, and through highlighting gaps in the research evidence, help to identify future research areas. ${ }^{22}$ Knowledge synthesis itself is not a new concept ${ }^{23}$ but it typically refers to the integration of various types of efficacy evidence. In healthcare, knowledge synthesis has principally focused on systematic reviews of interventions ${ }^{22}$ and not the totality of available knowledge regarding a treatment. We contend that interventions can be understood from, and evaluated against, a number of propositions, and that doing so is a total evidence and knowledge approach (TEKA). This includes but is not limited to a comprehensive description of the intervention in question, an understanding of the context in which the treatment will be employed, and the collective patient evidence. Further, employing such knowledge can help us to modify treatment and implementation plans to account for such evidence. It may even have the potential to override supporting research evidence if, for example, issues of treatment adherence and acceptability are apparent. 
We acknowledge the growing attention within the field to the role of implementation science, including knowledge translation strategies which seek to address the processes and outcomes of efforts to bridge the knowledge-practice gap. Implementation science includes the methods and strategies that might influence how EBP is adopted by clinicians and organisations, and requires consideration of implementation contexts. ${ }^{24-26} \mathrm{We}$ propose in this paper that independently of implementation processes and translation strategies, the evidence and knowledge which underpin interventions and which should influence clinical decisionmaking regarding implementation, themselves require a comprehensive examination as a crucial component of efforts to improve practice. The discussion presented here has been constructed to explore what those evidence sources or factors should include, and to demonstrate how this may be implemented in a systematic way.

\section{The benefits of practice and patient evidence}

The value of using efficacy evidence where it is available is not in dispute. Healthcare has the potential to become outmoded if the most current, up-to-date evidence is not incorporated into decision-making. ${ }^{27}$ However, as Sackett et al. ${ }^{5}$ argue, without due consideration of practice and patient evidence, our clinical practice risks becoming oppressed by research (efficacy) evidence. Clinical experience for example, can provide unique information and insights and offer an improved understanding of interventions, patient responses to individual treatments, and insights into contextual issues and constraints. Findings from a range of studies in the profession demonstrate that practice evidence is indeed important in the discipline's decision-making. ${ }^{28,29}$ Additionally, understanding patient groups' observations about an intervention also has the potential to provide further insights into commonly used interventions, including issues around adherence, usability and appeal. This in turn can provide clinicians with information on ways to improve the treatment 
TEKA

experience and adherence. Our understanding of practice and patient evidence at both individual and group levels remains considerably underdeveloped. This is especially so for systematically and rigorously acquired collective practice and patient evidence. ${ }^{30} \mathrm{We}$ ignore such evidence when appraising treatments to the detriment of reasonable and reasoned decision making and positive treatment outcomes. Retrieving and integrating such information makes common sense.

Current examples of evidence based and knowledge synthesis approaches in SLP

There has undoubtedly been progress made with regard to recognising the value and importance of practice and patient evidence, as well as improving accessibility to it. Such progress is evident in efforts to promote shared decision making ${ }^{31}$ and research in patient public involvement strategies. ${ }^{32}$ The work of the James Lind Alliance on aphasia is an example of this. ${ }^{33}$ The experience of individuals with communication impairments is also well described, in for example, developmental speech disorders, ${ }^{34,35}$ developmental language disorders, ${ }^{36}$ and stuttering, ${ }^{37}$ with clear illustrations of the lived experiences and impacts of such communication disorders. In SLP, there are known databases and tools available, which enable SLPs to access evidence on interventions, such as ASHA's Evidence Maps ${ }^{38}$ and in the UK, the Communication Trust's What Works ${ }^{39}$ database of interventions for childhood communication disorders. ASHA's Evidence Maps provide resources to support evidence informed practice. These evidence maps present recent publications relevant to each domain ('external scientific evidence', 'clinical expertise' and 'client perspectives') across a comprehensive range of disorders as well as professional roles (assessment, screening, service delivery and direct treatment interventions). What Works is a synthesis of knowledge across a systematic review of evidence, surveys of clinicians, and investigations of the preferred outcomes of children and their parents. ${ }^{41}$ While such databases have limitations, 
they are clear examples for SLPs of the benefits of employing a range of information based on the three pillars of EBP. Both the understanding generated in terms of areas of convergence and difference among the three sources of knowledge, and the dynamic nature of the databases, represent important examples of how we might begin to build more comprehensive epistemological frameworks to support clinical decision-making.

\section{Evidence and knowledge not explicitly recognised in EBP}

The explicit incorporation of knowledge not formally recognised in the EBP model is also advocated. Such information includes contextual evidence, which has been shown to be a factor in treatment decision-making both in speech-language patholog $y^{8}$ and other health disciplines such as nursing ${ }^{41}$ and which has been advocated by a number of authors. ${ }^{42,43}$ Even so, despite its impact, such information is not often explicitly stated when forming treatment decisions. Research in speech disorders has contributed to our understanding of service delivery and contextual evidence. ${ }^{44,45}$ Highlighting the benefits of a TEKA approach, these latter studies draw attention to mismatches between client expectations and experiences of intervention, and indeed, between the models and intensity of intervention suggested by empirical literature and actual service delivery experiences. Integrating this type of knowledge combined with other information on intervention implementation - for example, fidelity of intervention in a phonological treatment programme, ${ }^{46}$ is a critical reminder for researchers of the gap between researcher-designed interventions (where evaluated in a research context) and the practical demands of the clinical setting.

Beutow and Kenealy ${ }^{47}$ have also argued for additional forms of evidence, including judicial evidence and ethics-based evidence; it is remarkable how little information is available regarding such issues in the literature with reference to specific interventions. These include, for example, impacts such as treatment burden - defined as the patient workload 
resulting from an intervention and the impact on patient functioning and well-being. ${ }^{48}$ Incorporating such factors in a synthesised evidence and knowledge approach is consistent with the valuing of person-centred healthcare and shared decision making. Helping patients participate in their own healthcare decisions depends on high-quality information from a range of valid sources. If patients are not truly informed, as Elwyn ${ }^{49}$ notes, they will be unable to assess what is important to them and to generate truly informed preferences. Additionally, explicit consideration of alternative treatment options is also proposed in TEKA, as it is deemed to be integral to a comprehensive evaluation of an intervention. Such deliberation facilitates patient choice, contemplation of other possible treatment avenues, and explicit treatment comparisons, in addition to helping match treatments to the individual patient's needs. Further, it can serve to waylay concerns about habitual, traditional or cultural treatment selections in speech-language pathology. $8,29,50$

Finally, irrespective of other treatment knowledge, we contend that a clinician could reasonably judge the validity, scientific nature or reasonableness of an intervention, by either retrieving, or formulating, a clear description of that treatment, as outlined by Creaghead for example. ${ }^{51}$ This includes properties such as the theoretical proposition supporting the intervention and measures used to evaluate efficacy. Such an exercise encourages the clinician to engage explicitly with treatment components and rationales and to demonstrate a clear understanding of the treatment in question. Despite the importance of this, therapy descriptions in the literature are often unclear and underdeveloped. Ludemann et al. ${ }^{52}$ for example, investigated the adequacy of intervention descriptions in the SLP literature and found that no interventions were completely described either in the primary publications or from information retrieved from secondary locations. Such poor reporting of therapy descriptions has major implications for understanding, using, explaining, replicating and validating treatments. Further, such deficits in the literature do not encourage clinical SLPs to 
TEKA

spend time exploring the characteristics, merits and deficiencies of treatments. There are some examples of research contributing to our knowledge in this area. Interventions for phonological disorders in childhood for example, have been generally well-described in terms of treatment theory including mechanisms of action, ${ }^{3}$ and Wren et al. ${ }^{11}$ have mapped details of intervention components. Surveys of clinical practice and clinical decision-making such as that conducted as part of the Better Communication Research Programme (BCRP) ${ }^{54}$ have provided clinician reports (practice evidence) on intervention approaches, models, theories, and targets of intervention, including for phonological difficulties. ${ }^{55}$ Such evidence should encourage SLPs to actively engage with understanding interventions. We propose that SLPs prioritise conducting a treatment description exercise as a first TEKA step, given its importance in generating an understanding of core intervention characteristics. Spending time on formulating a full description of a treatment including for example, explicating the treatment theory and identifying how (and if) outcomes are measured, will facilitate SLPs considerably in evaluating individual treatments.

\section{A total evidence and knowledge approach (TEKA)}

The TEKA framework advocates using a broad range of evidence and knowledge as outlined in Table 1, to support the evaluation of SLP treatments and provide evidence for clinical decisions. This includes that which is advocated in the three pillars of EBP - although with extended definitions of practice and patient evidence - but also refers to other knowledge forms not incorporated by EBP but delineated in Table 1 and discussed in the previous text. It is our contention that complete and realistic treatment evaluations require more than efficacy, patient and practice evidence. TEKA thus raises an important central question, that is, what do we define as evidence and knowledge when evaluating treatments? There are two constituents integral to answering this question: to outline the various forms 
TEKA

that evidence and knowledge can take to assist in this process, and; to encourage the appraisal of knowledge in terms of validity or applicability, so that knowledge is not incorporated into evaluations and decision making uncritically.

The evaluation of interventions can be carried out in two main stages. The first part is an overall evaluation of a unique intervention based on TEKA domains (Table 1) without reference to individual patients. This stand-alone exercise can be updated on a predetermined basis to account for new information. Individual patient evidence can be integrated when required with this core information, to facilitate individualised decision-making. Thus, the TEKA model proposes the following steps:

1. Identify the intervention to be evaluated.

2. Gather evidence and information for each of the domains highlighted in Table 1.

3. Appraise the evidence and information retrieved for each knowledge form or domain where appropriate, using for example, published tools for research evidence. Sample questions have been provided in Table 2 to facilitate critical appraisal of information retrieved for the treatment description exercise. Consensus formation within SLP groups may also be useful for critically evaluating information retrieved.

4. Summarise the evidence for the intervention according to the domains highlighted in Table 1 and plot on a text document or graphic document such as a mind map.

5. When making a decision with regard to an individual patient, apply relevant individual patient information alongside the TEKA information, to facilitate clinical and shared decision-making.

Table 1 outlines the knowledge forms under discussion and the specific TEKA components for the intervention evaluation.

[insert table 1 here] 
TEKA

\section{Evidence and knowledge appraisal}

Whilst advocating for the inclusion of multiple forms of knowledge to produce integrated, reasoned, and reasonable decision-making, the authors fully acknowledge the potential demands of such an exercise, including the need to evaluate the reliability of the knowledge incorporated. This proposition regarding knowledge gathering and management is not a call to be less scientific, but to be more rigorous by engaging with a multiplicity of knowledge and to evaluate the validity of all retrieved knowledge. This model explicitly places the clinician at the centre of decision making and treatment evaluation, rather than delegating it to external agencies (i.e., through assigning primacy to efficacy evidence). It invites SLPs to take responsibility by engaging critically, proactively, and reasonably in the evaluation and employment of therapies and techniques.

This task appears relatively straightforward for efficacy evidence given the development of hierarchies of evidence and critical appraisal tools. Appraisal of research evidence often yields insights regarding research quality, stringency of protocols, the nature of target populations, highly restricted participant criteria (which may not reflect clinical populations), biases and potential vested interests. Even decision support tools such as clinical practice guidelines require critique. A recent review of international stroke guidelines for example, identified poor, old and sometimes inappropriate evidentiary bases for recommendations regarding the dysphagia intervention of thickened liquids. ${ }^{56}$ Thus, a critical, rather than accepting approach to all retrieved knowledge, is essential in order to facilitate improved decisions. In essence, critical thinking in speech-language pathology, as advocated by Finn, ${ }^{57}$ is at the heart of treatment evaluations. Critical thinking incorporates both skills and dispositions and, as such, reflects not just SLPs ability to engage critically, but also their desire to employ higher order thinking skills. As Wade et al. ${ }^{58}$ define it: "Critical thinking is the ability and willingness to assess claims and make objective judgments on the 
TEKA

basis of well-supported reasons and evidence rather than emotion and anecdote" (p 6-7). Thus, as Finn ${ }^{59}$ observes, clinicians require well-developed dispositions toward and competency in critical thinking, to ensure adoption and judicious use of evidence and knowledge.

\section{Collective practice and patient evidence}

Knowledge appraisal applies to all forms of retrieved knowledge including practice evidence, which incorporates individual and group clinical opinion and experience. While often informative, opinion-based knowledge has the potential to be prone to confirmation bias $^{60}$ and cannot be assumed to be highly rigorous, being judgment-based and therefore naturally subject to variability. This is one reason for promoting a collective voice approach to gathering practice (and patient) evidence per Table 1. In this approach, the collective experiences of individuals (clinicians and patients) who have used a specific intervention are gathered through research, or reviewed by engaging systematically with the literature. Clinicians can retrieve this type of patient evidence for example, by implementing a system of recording individual patient experiences regarding an intervention and then combining this evidence to form the collective voice. In employing the collective voice approach, we build substance, promote, and validate practice and patient knowledge; by publishing it we ensure that it is available in the same arena as efficacy evidence and that it is conferred with similar attention and consideration. Further, in the context of multiple examples of disharmony between research and practice, including the use of poorly empirically supported interventions, culturally-based treatments, and eclectic approaches to treatment, ${ }^{1,8,50}$ explicitly retrieving the practice reasoning for such interventions should lead to an improved ability to validate (or not) clinical practice/practice evidence. 
TEKA

\section{Treatment description questions}

As previously noted, an often-underused means of evaluating treatment integrity is through an intervention description exercise. The literature provides direction regarding fundamentals in describing interventions. ${ }^{51,59-62}$ Asking targeted questions such as those outlined in Table 2 can yield important and insightful knowledge and highlight gaps in information and logic. Thus, while the act of knowledge retrieval (i.e., learning/reading about a new treatment) is important, any treatment evaluation exercise can be considered poorly and incompletely performed if the knowledge retrieved is not also appraised. Sample questions that can be addressed through this exercise are highlighted in Table 2 and an example of a treatment description has been provided in Appendix 1.

[insert table 2 here]

\section{TEKA example}

This paper calls for reasonably informed treatment decision-making and proposes what such knowledge might constitute. One example is presented to demonstrate what using such an approach might produce in terms of intervention information. The example relates to the bolus modification intervention of thickened liquids/fluids (TL) for a hypothetical patient (Johnny G) with dysphagia and aspiration post-stroke. For this intervention, relevant evidence and knowledge across the TEKA domains was retrieved and this is summarised in Table 3. The retrieved information, including details of supporting references are provided in Appendix 1.

[insert table 3 here]

The TEKA exercise for this intervention resulted in a sizeable volume of information as demonstrated in Table 3 and Appendix 1. Such information when combined with the SLPs 
TEKA

knowledge of a patient, would enable the clinician to form a reasonable, patient-centred, knowledge-based plan for management of the case in question. The exercise has served to reveal treatment strengths and challenges, encouraged engagement with treatment theory while highlighting knowledge gaps and knowledge certainties. Despite the limited research evidence base for using TL, it is known that $78 \%$ of dysphagia therapists generally, and $97 \%$ of SLPs working with people with dysphagia post-stroke employ this treatment. ${ }^{63,64}$ This exercise emphasizes how using multiple forms of evidence and knowledge encourages SLPs to explicitly engage in and rationalise clinical decision making. Using TEKA could help to facilitate reasonable judgements irrespective of whether SLPs choose to employ the intervention in question or not.

\section{Applying TEKA}

The authors are not suggesting TEKA is a simple exercise, nor do we underestimate the effort involved in applying such a model. It is likely to be time intensive particularly in the initial stages of an intervention evaluation. However, EBP itself has not proved to be an easy or apposite answer, and better models to support decision-making are still required. We appreciate that time is a scarce commodity for practicing clinicians, that limited time impacts service delivery for $\operatorname{SLPs}^{65-67}$ and that there is an inherent cyclical problem (clinicians don't have time for this, but time is one of the reasons their treatment may not be effective). A worthwhile treatment evaluation cannot be accomplished without effort but will produce long-term clinical guidance and impact including regarding service delivery and treatment outcomes. It is certainly important for our discipline that we engage deeply with our treatment tools, that we can rationalise our treatment choices and defend our decisions based on evidence and knowledge. This is true whether they are empirically supported or not, but especially so in cases where the efficacy evidence is lacking. In a profession wedded to 
TEKA

scientific principles, SLPs should be engaged in developing argumentation regarding the treatments and techniques they use in practice. The suggestions below are intended to facilitate engagement with TEKA or similar exercises while acknowledging the time constraints of clinicians.

1. SLPs can utilize shortcuts to commence this exercise by accessing components already completed, such as systematic reviews in the literature, or through work already available on SLP websites such as ASHA Evidence Maps ${ }^{38}$ and What Works. ${ }^{39}$ This can be added to by noting and plugging the gaps in knowledge observed.

2. Academics and researchers can conduct TEKA exercises with students SLPs either in specific modules or as a team research project. Student pairs/groups can carry out individual elements of TEKA such as collecting or synthesising the patient evidence or conducting a treatment description exercise. The products of these individual exercises can be combined to generate a full treatment evaluation or TEKA map. New student cohorts can incorporate updates and additions from year to year. Dissemination of information generated by students should be encouraged, so that TEKA treatment evaluations benefit the larger SLP discipline. Dissemination can occur in variety of ways including via institutional repositories, conference presentations and posters, publications and workshops.

3. SLPs can prioritise one element of the TEKA exercise at a time for example, the treatment description exercise, thus building the knowledge base over time. Alternatively, groups of regional SLP teams can agree which treatment requires evaluation, with each team responsible for carrying out one part of the TEKA exercise. The whole group can then combine their efforts to formulate a 
comprehensive treatment evaluation and build a TEKA map. As for the students in point 2, this can be shared via various arenas with other SLPs.

4. Clinicians can incorporate key TEKA aspects by explicating their client specific and theoretical knowledge. In clinical practice, much of the thinking processes become implicit - this applies not just to the knowledge underlying clinical decisions but to the reasoning supporting decisions as well. Treatment evaluations would be well supported by explicating both treatment and patient knowledge and by mapping this knowledge onto TEKA headings.

5. We recommend building TEKA into existing activities and tasks to which clinicians already devote time (e.g. supervision/mentoring meetings, team meetings, training/CE sessions), rather than creating a new forum within which it will be discussed. Additionally, clinicians who report on a training event or who review a journal article to feedback to colleagues, could incorporate the TEKA questions in their review of the training or article. For example, what was learned about the intervention's mechanism of action; what was learned about clients'/research participant's experiences of this intervention; what are the issues we need to consider in employing this intervention with our client/caseloads. Clinical teams could present worked case examples using the template provided here. This could generate valuable reflection on the processes and application of this more holistic evidence-based planning approach, and facilitate increased confidence for individuals to embed this more widely.

6. Partnering between clinicians and researchers could be beneficial in the sharing and combining of knowledge to co-produce a TEKA map for an intervention. This would play to each group's strengths. Clinicians could, for example, very usefully inform the research community about those aspects of interventions that are not being described or captured in published empirical research. These may include client experiences of 
an intervention and contextual evidence. As noted previously, they could also formally collate this information when delivering services to build the collective voice. Researchers and educational institutions could share newest empirical information, search results or systematic review summaries.

\section{Conclusion}

SLP researchers and clinicians need to explicitly employ a more comprehensive approach to intervention evaluation, such as that outlined in this paper in the form of TEKA. This includes extended interpretations of two of the three core elements that are advanced in the EBP model of which practice and patient evidence, we argue, are defined inadequately. It also includes but is not limited to, a comprehensive description of the intervention in question, an understanding of the context in which the treatment will be employed, and judico-ethics evidence. Employing such knowledge can help us to rationalise treatment selections and modify treatment and implementation plans to account for such evidence. EBP is a fairly one-dimensional way of evaluating interventions and a top-down construct, which overestimates the role of efficacy evidence in clinical decision making and intervening. It is incumbent upon the discipline to look beyond the traditional model of EBP. A broader range of retrieved knowledge, representing a more grounded clinical understanding of intervention, should be incorporated more overtly into our intervention evaluations and clinical decisionmaking.

Complexity in using an approach such as TEKA will depend on the area of practice and the specific intervention under examination. Even in some relatively well-developed arenas (for example developmental speech disorders), whilst available evidence across interventions can now inform many of the elements of the framework, it is challenging to find an example of an intervention for one area of need, with information across each of the 
TEKA

knowledge forms identified in Table 1. In applying TEKA, though, SLPs must also be careful to avoid what Strauss ${ }^{68}$ calls the knowledge translation imperative, that is, that all knowledge must be translated into action. Instead, we need to ensure that there is a mature and valid reflection on the knowledge retrieved, prior to treatment decisions being enacted at the individual and service levels.

A TEKA approach may provide some answers which have not yet been delivered by EBP and help allay fears of a reductionist approach to practice. ${ }^{22}$ TEKA acknowledges that there are multiple forms of legitimate evidence and knowledge and promotes reasonably informed clinical and shared decisions which are grounded in the totality of evidence and knowledge. It allows for the recognition of and open response to contextual issues and has the potential to be more explicitly patient-centric. A true synthesis approach such as that outlined here, can help reduce what Farr and Cressey ${ }^{69}$ describe as the tensions that exist between patient-centred, relational care and the pressures of efficiency and rationalisation. Moreover, it fosters clinical ownership of and academic/clinical partnerships in treatment evaluations, rather than delegating it to external forces - specifically efficacy evidence - and encourages us to focus on integration rather than implementation. This approach responds to calls by Greenhalgh and Maskrey ${ }^{2}$ who advocate for "real" EBP, which is characterised by expert judgment rather than mechanical rule following and includes making the ethical care of the patient its top priority. As they argue, "practitioners, together with their patients, must be free to make appropriate care decisions that may not match what "best (average) evidence" seems to suggest" ( $(\mathrm{p} 3)$. In doing so, clinicians must also be able to demonstrate clearly that they have arrived at those decisions in a logical, informed way.

Adopting an approach such as TEKA may contribute towards more effective practice and optimal outcomes for the clients of speech-language pathology services. This would require the combined inputs and best efforts of both researchers and clinicians. TEKA makes 
TEKA

logical sense and compels us to examine our decision-making. It can provide added legitimacy to clinical decisions in the presence or absence of research evidence for an intervention. In effect, it aligns clearly with Sackett's ${ }^{3}$ call to be "conscientious, explicit and judicious" ( $\mathrm{p} 71)$ in making decisions about the care of individual patients.

\section{Acknowledgments}

Thanks to Patrick Finn, Professor of Communications Sciences and Special Education at the University of Georgia, Athens for his help in reviewing this paper.

\section{References}

1. McCurtin A, Roddam H. Evidence based practice: Opportunities for growth or professionals under siege. Int J Lang Commun Disord 2012;47(1):11-26.

2. Greenhalgh T. How to Read a Paper: The Basics of Evidence-Based Medicine. 1997; London: BMJ Books.

3. Reilly S, Douglas J, Oates J. Evidence based Practice in Speech Pathology. 2004; London: Whurr.

4. Mykhalovskiy E, Weir L. The problem of evidence based medicine: directions for social science. Soc Science Med 2004;59:1059-1069.

5. Sackett DL, Rosenberg, WMC, Gray JAM, et al. Evidence-based medicine: what it is and what it isn't. BMJ 1996;312:71-72.

6. Dollaghan CA. The Handbook for Evidence-based Practice in Communication Disorders. 2007; Baltimore, MD: Paul H Brookes.

7. https://www.asha.org/Research/EBP/. Accessed June $27^{\text {th }} 2019$. 
8. McCabe PJ. Elizabeth Usher Memorial Lecture: How do we change our profession? Using the lens of behavioural economics to improve evidence-based practice in speech-language pathology. Int J Speech-Lang Pat 2018;20(3):300-309.

9. Greenhalgh T, Maskrey N. Evidence based medicine: a movement in crisis? BMJ 2014;4:348. Available at: https://doi.org/1010.1136/bmj.g3725. Accessed January 19 2019.

10. Stephens D, Upton D. Speech and language therapists' understanding and adoption of evidence-based practice including commentaries by Erickson S and McCurtin A. Int J Ther Rehab 2012;19(6):333-334.

11. Wren Y, Harding S, Goldbart J, et al. A systematic review and classification of interventions for speech sound disorder in preschool children. International Journal of Language and Communication Disorders 2018; 53(3):446-467.

12. Law J, Garrett Z, Nye C. Speech and language therapy interventions for children with primary speech and language delay or disorder (Review). Cochrane DB Syst Rev 2003;3:CD004110.

13. Roulstone S. Evidence, expertise, and patient preference in speech-language pathology. Int J Speech-Lang Pat 2011;13(1):43-48.

14. Hubble MA, Duncan BL, Miller SD. Introduction. In: Hubble MA, Duncan BL, Miller SD, eds. The Heart \& Soul of Change: What Works in Therapy. Washington DC: American Psychological Association; 1999.

15. Moerman DE. Meaning, Medicine and the Placebo Effect. Cambridge: Cambridge University Press; 2002.

16. Plesk PE, Greenhalgh T. The challenge of complexity in health care. BMJ 2001;323:625-628. 
17. McCurtin A, Clifford A. What are the primary influences on treatment decisions? How does this reflect on evidence-based practice? Indications from the discipline of Speech \& Language Therapy. J Eval Clin Pract 2015;21:1178-1189.

18. Law J. Evidence-based practice and its application to developmental language disorders. In: Law J, McKean C, Murphy CA, et al eds. Managing Children with Developmental Language Disorder. Theory and Practice across Europe and Beyond. London: Routledge Taylor and Francis; 2019: 6-29.

19. Murphy CA. The limits of evidence and the implications of context: considerations when implementing pathways to intervention for children with language disorders. Int J Lang Comm Dis 2019;54(1):20-23.

20. Satterfield JM, Spring B, Brownson RC, et al. Toward a transdisciplinary model of evidence-based practice. Milbank Q 2009;87:368-390.

21. Berg H, Slaattelid R. Facts and values in psychotherapy: A critique of the empirical reduction of psychotherapy within evidence-based practice. J Eval Clin Pract 2017;23(5):1075-1080.

22. Kastner M, Tricco AC, Soobiah C et al. What is the most appropriate knowledge synthesis method to conduct a review? Protocol for a scoping review. BMC Med Res Methodol 2012; 12:114.

23. Tricco AC, Tetzlaff J, Moher D. The art and science of knowledge synthesis. J Clin Epidemiol 2001;64: 11-20.

24. Titler MG, Everett LQ, Adams S. Implications for Implementation Science. Nurs Res 2007;56:53-59.

25. Olswang LB. Prelock, PA. Bridging the Gap between Research and Practice: Implementation Science. J Speech Lang Hear Res 2015;58:s1818-s1826. 
26. Douglas NF, Burshinc VL. Implementation Science: Tackling the Research to Practice Gap in Communication Sciences and Disorders. Perspectives of the ASHA Special Interest Groups, 2019;4:4-7.

27. Siminoff LA. Incorporating patient and family preferences into evidence-based medicine. BMC Med Inform Dec 2013;13(Suppl 3):S6. Available at: http://www.biomedcentral.com/1472-6947/13/S3/S6. Accessed September 92018.

28. Mackenzie C, Muir M, Allen C. Non-speech oro-motor exercise use in acquired dysarthria management: regimes and rationales. Int J Lang Comm Dis 2010;45(6):617-629.

29. McCurtin A, Carter B. We don't have recipes; we just have loads of ingredients. Explanations of evidence and clinical decision-making by speech \& language therapists. J Eval Clin Pract 2015;21:1142-1150.

30. McCurtin A, Healy C, Kelly L, et al. Plugging the patient evidence gap: what patients with swallowing disorders post-stroke say about thickened liquids. Int J Lang Comm Dis 2018;53(1):30-39.

31. Stiggelbout AM, Van der Weijden T, De Wit MPT, et al. Shared decision making: really putting patients at the centre of healthcare. BMJ 2012;344:e256. Available at: https://doi.org/10.1136/bmj.e256. Accessed January 42019.

32. MacFarlane A, Galvin R, O’Sullivan M, et al. Participatory methods for research prioritization in primary care: an analysis of the World Café approach in Ireland and the USA. Family Practice 2017;34(3):278-284.

33. Franklin S, Harhen D, Hayes M, et al. Top 10 research priorities relating to aphasia following stroke, Aphasiology 2018. 32:11, 1388-1395. 
34. McCormack J, McLeod S, McAllister L, et al. My Speech Problem, Your Listening Problem, and My Frustration: The Experience of Living with Childhood Speech Impairment. Lang, Speech Hear Ser 2010;41(4):379-392.

35. McCormack J, McAllister L, McLeod S, et al. Knowing, having, doing: The battles of childhood speech impairment. Child Lang Teach The 2012;28(2):141-157.

36. Lyons R, Roulstone S. Well-Being and Resilience in Children with Speech and Language Disorders. Journal of Speech, Language, and Hearing Research 2018:61:324-344.

37. Connery A, McCurtin A, Robinson K. The lived experience of stuttering: a synthesis of qualitative studies with implications for rehabilitation. Disability and Rehabilitation 2019. Available at: https://doi.org/10.1080/09638288.2018.1555623. Accessed April $14,2019$.

38. American Speech Language Hearing Association Evidence Maps. https://www.asha.org/evidence-maps/. Accessed June 26 2019.

39. Communication Trust: What Works? Available at: http://www.thecommunicationtrust.org.uk/whatworks. Accessed March 10, 2019.

40. Law J, Roulstone S, Lindsay G. Integrating external evidence of intervention effectiveness with both practice and the parent perspective: development of 'What Works' for speech, language, and communication needs. Dev Med Child Neurol 2015;57(3):223-228.

41. Parahoo K. Barriers to, and facilitators of, research utilization among nurses in Northern Ireland. J Adv Nurs 2000;31(1):89-98.

42. Hoffmann T, Bennett S, Del Mar C. Evidence-Based Practice across the Health Professions-E-Book. Elsevier Health Sciences/Churchill Livingstone Australia; 2013. 
43. May C. Towards a general theory of implementation. Implementation Science 2013;8:18. Available at: https://doi.org/10.1186/1748-5908-8-18. Accessed July 10 2018.

44. McAllister L, McCormack J, McLeod S, et al. Expectations and experiences of accessing and participating in services for childhood speech impairment. Int J SpeechLang Pat 2011;13(3):251-267.

45. Ruggero L, McCabe P, Ballard KJ, et al. Paediatric speech-language pathology service delivery: An exploratory survey of Australian parents. Int J Speech-Lang Pat 2012;14(4):338-350.

46. McCormack J, Baker E, Masso S, et al. Implementation fidelity of a computerassisted intervention for children with speech sound disorders. Int J Speech-Lang Pat 2017;19:266-277.

47. Beutow S, Kenealy T. Evidence-based medicine: the need for a new definition. J Eval Clin Pract 2000;6(2):85-92.

48. Gallacher K, Jani, B, Morrison D. et al. Qualitative systematic reviews of treatment burden in stroke, heart failure and diabetes - Methodological challenges and solutions. BMC Med Res Methodol 2013;13:10. Available at: https://doi.org/10.1186/14712288-13-10. Accessed July 102018.

49. Elwyn G, Frosch D, Thomson R, et al. Shared Decision Making: A Model for Clinical Practice. J Gen Intern Med 2012;27(10):1361-1367.

50. Kamhi AG A Meme's Eye View of Speech-Language Pathology. Lang Speech Hear Ser 2004;35:105-111.

51. Creaghead N. Evaluating language intervention approaches: contrasting perspectives. Lang Speech Hear Ser 1999;30(4):335-338. 
52. Ludemann A, Power E, Hoffman TC . Investigating the Adequacy of Intervention Descriptions in Recent Speech-Language Pathology Literature: Is Evidence From Randomized Trials Useable? Am J Speech-Lang Pat 2017;26:443-455.

53. Rvachew S, Brosseau-Lapre F. Developmental Phonological Disorders Foundation of Clinical Practice. San Diego CA: Plural;2012.

54. Better Communication Research Programme. Available at: https://www.gov.uk/government/collections/better-communication-researchprogramme. Accessed March 10, 2019.

55. Roulstone S, Wren Y, Bakopoulou I, et al. Exploring interventions for children and young people with speech, language and communication needs: A study of practice. Available at: https://www.gov.uk/government/uploads/system/uploads/attachment_data/file/219627 /DFE-RR247-BCRP13.pdf. Accessed July 112018.

56. McCurtin A, Boland B, Kavanagh M, et al. A systematic review of Stroke Clinical Practice Guidelines and the intervention of thickened liquids for aspiration: Do recommendations support evidence based decision-making? BMC Med Inform Decis 2019; in press.

57. Finn P. Critical Thinking: Knowledge and Skills for Evidence-Based Practice. Lang Speech Hear Serv School 2011;42:69-72. Available at: https://doi.org/10.1044/01611461(2010/09-0037). Accessed July 32018.

58. Wade C, Tavris, C, \& Garry M. Psychology, 11th ed. NJ: Prentice Hall; 2014.

59. Finn P. Establishing the validity of stuttering treatment effectiveness: The fallibility of clinical experience. Perspect Flu Flu Disord 2004;14:9-12.

60. Finn P, Bothe AK, Bramlett RE. Science and pseudoscience in communication disorders: Criteria and applications. Am J Speech-Lang Pat 2005;14:172-186. 
61. Turkstra LS, Norman R, Whyte J, et al. Knowing What We're Doing: Why Specification of Treatment Methods Is Critical for Evidence-Based Practice in Speech-Language Pathology. Am J Speech-Language Pat 2016;25(2):164-171.

62. Vyse S. Where do fads come from? In: Jacobson JJ, Foxx RM, Mulick JA, eds. Controversial Therapies for Developmental Disabilities: Fad, Fashion, and Science in Professional practice NJ: Lawrence Erlbaum Associates; 2005; 10-15.

63. McCurtin A, Healy C. Why do clinicians choose the therapies and techniques they do? Exploring clinical decision making via treatment selections in dysphagia practice. Int J Speech-Lang Pat 2017;19(1):69-76.

64. Jones O, Cartwright J, Whitworth A, et al. Dysphagia therapy post stroke: an exploration of the practices and clinical decision-making of speech-language pathologists in Australia. Int J Speech-Lang Pat 2018;20920:226-237

65. Biancone TL, Farquharson K, Justice LM et al. Quality of language intervention provided to primary-grade students with language impairment. J Comm Disord 2014;49:13-24.

66. Katz LA, Maag A, Fallon KA et al. What makes a caseload (un) manageable? Schoolbased speech-language pathologists speak. Lang Speech Hear Serv School 2010;41(2):139-51.

67. Pavelko SL, Owens Jr RE, Ireland M. Use of language sample analysis by schoolbased SLPs: Results of a nationwide survey. Lang, Speech Hear Serv Schools 2016:47(3):246-258.

68. Straus SE, Tetroe JM, Graham ID. Knowledge translation is the use of knowledge in health care decision making. J Clin Epidemiol 2011;64(1):6-10. 
TEKA

69. Farr M, Cressey P. Understanding staff perspectives of quality in practice in healthcare. BMC Health Serv Res 2015;15:123 Available at: https://doi.org/10.1186/s12913-015-0788-1. Accessed November 122018.

Learning outcomes: As a result of this activity, the reader will be able to (1) Identify the various forms of knowledge and evidence which underpin comprehensive intervention evaluations; (2) Understand the importance of retrieving multiple forms of knowledge and evidence when evaluating interventions and forming clinical decisions;(3) Understand how individual forms of knowledge can contribute both uniquely and in combination to a better and broader understanding of an intervention, and; (4) Recognize the importance of critical thinking and knowledge appraisal in undertaking treatment evaluations.

\section{CEU Questions \& Answers}

1. Which of these forms of evidence is not in the original definition of evidence based practice $(\mathrm{EBP})$ ?
a. Clinician experience
b. Efficacy evidence
c. Contextual evidence
d. Patient values and experience
e. All of the above

2. Which non-efficacy evidence and knowledge might we consider when conducting intervention evaluations and forming clinical decisions?
a. Judico-ethics evidence
b. Contextual evidence
c. Collective practice evidence 
d. Collective patient evidence

e. All of the above

3. A clinician should be able to reasonably judge the validity or reasonableness of an intervention by:
a. Checking if other SLPs are using it
b. Accepting the therapy originators' position
c. Performing a treatment description exercise
d. Asking a colleague's opinion

4. Collective patient or practice evidence refers to which of the following:
a. Individual opinion
b. Individual experience
c. The experience and perceptions of a group of people familiar with the intervention in question and retrieved from either conducting or synthesizing the research
d. The opinions and experiences of colleagues and friends

5. SLPs are central to the judicious and comprehensive evaluation of the interventions they employ. To perform as such they must:
a. Accept other clinician's opinions
b. Act as critical thinkers
c. Follow authority figures
d. Utilize efficacy evidence without reference to other forms of knowledge and evidence

\section{Answer Key}

1. c

2. $\mathrm{e}$ 
3. c

4. c

5. b

Appendix 1: Information retrieved relevant to components of knowledge management, regarding use of thickened liquids (TL) for aspiration for people with dysphagia (PWD) post-stroke and for hypothetical patient Johnny G.

\section{Description of therapy}

The TL intervention is an established bolus modification treatment used to prevent/reduce aspiration in people with dysphagia. It comes in various forms including prethickened drinks but is most typically given by adding a thickening agent (starch or gum based) in powder form to regular drinks. Drinks can be thickened to various consistencies (based on national/international descriptors) appropriate to the individual's needs, as determined by a speech-language pathologist (SLP) after clinical/instrumental assessment. It is designed to slow bolus speed and thus increase swallow safety by minimising aspiration risk. ${ }^{70,71}$ Slowing bolus speed, theoretically means that the bolus is retained in the oral phase of the swallow for longer, that there is more time to organise/ initiate the swallow, and that the bolus should move through the pharyngeal phase more slowly. This implies that individuals with delayed or disorganised swallows resulting in penetration or aspiration would benefit most from this intervention. It would also lead to the question as to whether individuals with oral stage disorders (e.g. problems moving the bolus anteriorallyposteriorally) might find a thicker consistency more difficult. Candidacy tends to be decided on an individual basis; ideally this is done using instrumentation (i.e. videofluroscopy, VFSS, or fibreoptic endoscopic examination of swallowing, FEES). Newman et al. ${ }^{71}$ have called for 
TEKA

the development of highly specified candidacy criteria to "establish the optimal viscosity level for each phenotype of dysphagic patients" (p. 232).

Individual outcomes are best evaluated via instrumentation, specifically VFSS or FEES, which can provide pre and post-intervention evidence for individual cases. Other measures include negative findings, specifically reduction in coughing on drinking, and lack of or reduction in occurrence of aspiration (difficult to gauge in silent aspirators) and of aspiration pneumonia. For this population, TL is known to have side effects or risks. These include effects on oral and pharyngeal transit times, increases in oral and pharyngeal residue, and increased risk of dehydration. ${ }^{70-73}$ It may also be that the oral effort may be increased as muscles may need to work harder to move the thicker liquid. Further, thickened liquids are known to be poor at quenching thirst. They also result in poor flavour release, and effects of delayed dissolution and disintegration of some medications have been demonstrated with TL. ${ }^{74}$ Starch-based thickeners have been shown to contain their consistency with human saliva less well than gum-based thickeners ${ }^{75}$.

\section{Research evidence}

Recent systematic reviews (SRs) for this intervention are available and referenced below and these represent the highest level of efficacy evidence. Most evaluate the evidence for TL in people with dysphagia (PWD) for a range of conditions. Although the evidence base remains currently inadequate and often contradictory, ${ }^{71,73,76,77}$ the consensus from most recent systematic reviews is that there is some evidence for TL reducing the risk of airway invasion. ${ }^{71,73,78}$. Most of these reviews also conclude that the evidence in favour of TL is not strong. One meta-analysis ${ }^{77}$ concluded there was no significant difference in the risk of pneumonia in aspirating patients who took thin liquids with safety strategies, compared with those who took thickened liquids only. In an SR conducted specifically with regard to stroke, 
TEKA

Foley et al. ${ }^{79}$ concluded, consistent with most reviews, that TL is associated with a reduced risk of aspiration (in the acute stages of stroke) but strong research support is lacking. Most stroke clinical guidelines recommend TL either in isolation or as one element of a multicomponent treatment programme. These recommendations are often made using older, sometimes inappropriate, evidence and may be based on the consensus of the guideline development group. ${ }^{56}$

\section{Practice evidence}

$78 \%$ of dysphagia therapists generally and $97 \%$ of SLPs working with PWD poststroke use TL. ${ }^{63,64}$ It is ranked in the top three dysphagia interventions, and $85 \%$ of SLPs regard it as effective. ${ }^{63,80}$. This high use in the profession in the absence of clear supporting evidence suggests a cultural influence on use, which should be considered. However, it is likely that clinicians retrieve individual patient evidence via clinical observations and instrumental techniques to support administration of TL and without which it would not continue to be used to such a high degree. A recent qualitative study of 22 SLPs working with PWD post-stroke regarding the TL intervention ${ }^{81}$ found that TL was employed by SLPs as a first step on the treatment ladder and use pivoted on safety-first reasoning. SLPs also perceived that there were limited treatment alternatives for reducing the risk of aspiration. Both clarity and contradictions around TL use were revealed, with SLPs acknowledging multiple factors which impact treatment effectiveness including hospital, patient and product issues.

Own hypothetical clinical opinion, group consensus, known experts

"I have NUMBER years clinical experience working in acute settings with patients with dysphagia including those PWD post-stroke. I have used TL frequently to treat aspiration risk, and this has been the main treatment used in the hospital for aspiration. I 
TEKA

find TL generally effective especially in cases where patients need time to initiate a swallow, where there is no anterior spillage, and especially in the acute stages, when other dysphagia interventions may not be possible. However, given the limited access to instrumentation in this hospital I cannot always provide case evidence of treatment effect. Also, I am aware that there are other variables at play which may impact outcomes including general recovery. In addition, I know that many of my patients with stroke are discharged before I can assess outcomes so there are cases where I am unable to follow up and cannot evaluate how they have done on the treatment. I have discussed this with my colleagues, and we are generally agreed that it is a useful treatment in the acute stages, but we also appreciate that patients don't like it and there are treatment adherence issues. We have been trying other options recently including risk feeding. I have also been in contact with NAME, who is a national expert in the area, and she advises that individual patient clinical evidence, such as that retrieved from videofluroscopy or FEES, and the patient's opinion will be the best way to evaluate the treatment's effectiveness and usability."

Patient evidence

The TL treatment has been associated with discontent among PWD resulting from a range of conditions. ${ }^{82,83}$. Non-adherence rates with TL are reported to vary between $40 \%$ and $80 \% .{ }^{82,84}$ In Logemann et al.' ${ }^{85}$ large scale study, a sample of 711 PWD who trialled three aspiration interventions were shown to prefer non-TL-based treatments such as postural maneuvres and preferred to risk pneumonia than to use TL. Stroke-specific patient evidence is sparse. McCurtin et al. ${ }^{28}$ interviewed 14 community dwelling PWD post- stroke and found that participants disliked TL and this dislike impacted clinically in terms of adherence, hydration and quality of life. Lack of sensory appeal was important in framing patient dislike of the treatment. Participants' involvement in and understanding of reasons for prescription 
of TL was poor, leading to uncertainty regarding the treatment. The authors concluded that TL can be considered a burdensome treatment from multiple perspectives including product palatability, treatment uncertainty and treatment adherence issues. Two other studies ${ }^{86,87}$ included stroke patients as a subset in their samples with similar results. In the Swan et al. ${ }^{87}$ study, participants linked TL with a worse quality of life. This dissatisfaction is noted even among health professionals in the acute environment. Lim et al.'s ${ }^{88}$ healthcare utilities study for example, found that the health professions participants would prefer to sacrifice multiple years of their life instead of using TL.

Patient's opinion, values, preferences

"I (the clinician) discussed the treatment with the patient/partner in question. Johnny $G$ is in the acute stages post-stroke, has comprehension and expression difficulties and is not able to meaningfully participate in shared decision making at this stage. His wife explained that he isn't a big drinker generally. I explained the treatment options including TL. She felt that Johnny $G$ would be highly unlikely to drink a thickened drink and that she is concerned about him coughing and choking. She would like to "get him back to normal" and is happy to trial risk feeding as long as he is monitored closely. This decision will be reviewed tomorrow as his status improves."

\section{Contextual evidence}

$\underline{\text { Costs }}$

There is evidence which highlights the cost to the health services if a patient with stroke gets pneumonia from aspiration while in the hospital. These include longer hospital stays, increased cost, greater disability and poor nutrition while in the hospital. ${ }^{89}$ However, as $\mathrm{O}^{\prime} \mathrm{Keefe}^{90}$ argues, reduction in pneumonia resulting from TL remains unproven. There may also be additional costs involved which need to be considered including the costs 
TEKA

for treating dehydration and its consequences resulting from TL. ${ }^{90}$ For some patients, depending on the system, there is also a cost for the product itself. This varies from patient to patient, geography and length of time on the treatment. The monthly costs for American patients on TL is $\$ 174$ - \$289 while the annual cost to the NHS in England is circa $£ 14$ million per year. ${ }^{90,91}$

\section{Implementation}

Murray et al..$^{92}$ conducted a survey of health care professional prescribing and monitoring practices of TL. Respondents highlighted institutional factors such as attributing inadequate fluid intake to the hospital system (i.e., reporting that patients on TL are not offered enough thickened fluid or their consumption is not monitored closely enough). Some suggested that the patient's functional disabilities (immobility, poor fine motor control, communication and/or cognitive impairment) resulted in them being unable to open packages and access drinks independently. Mixing of TL to specified individual consistencies is often problematic and there is evidence that staff, including aides, nurses and SLPs, do not reliably mix consistencies ${ }^{80,93}$ Hospitalised individuals have also been found to have insufficient access to containers of liquids, difficulty opening drink containers, and difficulty accessing staff to assist them with drinking. ${ }^{74}$ Such findings raise concern about the impact of training of staff, the monitoring of preparation practices and treatment fidelity. Further, thickened drinks are sometimes not drunk quickly enough and thickener may dissolve and not be as effective.

$\underline{\text { Use of TL for other purposes }}$

Because patients on TL typically receive drinks solely via this means, thickened drinks can be used for other purposes including to achieve hydration and ingest medications. ${ }^{74,81}$ This often means the treatment is being used for other purposes and may impact on fidelity and safety. 
TEKA

Local/national contextual information here such as policy, resource constraints.

"It is standard practice in our hospital to use this treatment for all patients at risk of aspiration due to dysphagia. This practice is supported by the professional association's guidelines regarding treating aspiration. We do not have regular access to instrumentation such as VFSS and FEES so are unable to assess each patient individually at time of treatment commencement. While we provide training to staff and have notices with directions on consistencies above patient beds, staff turnover and general busyness often mean that guidelines are not adhered to. Additionally, jugs of unthickened water are frequently left at bedsides of patients requiring TL. If patients are discharged home while still requiring the treatment, we find this is often done without a prescription for TL or the knowledge of the SLPs. As a department, in the last 6 months we have been incrementally trialling risk feeding and are developing protocols with the nursing teams on the acute wards. We plan to discuss this option with patients going forward."

\section{Judico-ethics based evidence}

Thickeners are commercial products; thus, there are naturally vested interests in maintaining their use in healthcare. Empirical, clinical and patient rather than commercial interests should always be at the forefront of decision-making. Often informed consent for treatment is difficult to obtain in the acute stages of post-stroke, as capacity to give consent is reduced. Guidelines for obtaining informed consent including working with carers can be used for this purpose, with the issue revisited with the patient once capacity improves. Issues pertaining to patient impact need consideration, especially the principle of "do no harm", as patient evidence suggests that this treatment is disliked by individuals and has impact on quality of life and well as the potential to create treatment burden. ${ }^{28,87}$ There is a need to weigh treatment benefit against treatment burden for each individual. 
TEKA

Legal evidence where known

"I (the clinician) am not aware of any legal precedents regarding this treatment"

Other treatment options

SLPs perceive that there are limited treatment options for aspiration and that TL is the best available. ${ }^{81}$

Free water protocols (FWPs): FWPs are a possible option. Murray et al. ${ }^{94}$ found that they are only used $14 \%$ of the time in healthcare facilities. FWPs provide access to unthickened water but require strict oral hygiene protocols.

Chin tuck/down: This postural manoeuvre is well used in practice typically as part of an overall swallowing programme. ${ }^{63}$ The intent of postural techniques is to improve function and compensate for abnormalities that have not yet been rehabilitated. ${ }^{89}$ For these techniques, consistent with TL, there is limited evidence for their effectiveness and constraints such as the ability of the healthcare providers to ensure protocols and compliance. Fraser and Steele ${ }^{95}$ concluded that the use of chin down during teaspoon administration of a bolus with PWD poststroke should be avoided unless verified by VFSS. In their retrospective review of charts and VFSS exams of patients with neurogenic dysphagia, Socanato et al. ${ }^{96}$ found that the chin tuck should benefit dysphagic patients with delayed swallowing and reduced laryngeal elevation, and that the effectiveness of the chin tuck is related to the overall degree of dysphagia. They concluded that the more severe the dysphagia, the less effective the manoeuvre and that it is potentially more useful in 'mild dysphagia'

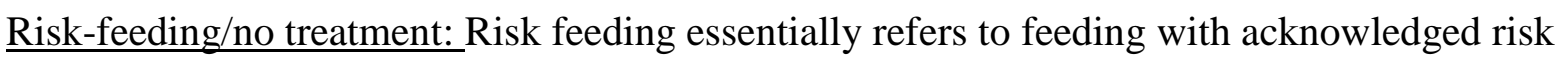
and implies an acceptance of the risks of oral feeding for that patient. Risk-feeding can take many forms varying with each individual case and the risks taken likewise vary based on each PWD. In some cases, the risks will be small or putative while in others they will be 
TEKA

great. According to Sommerville et al, ${ }^{97}$ the swallow is unlikely to improve on a risk-feeding regimen. With PWD post-stroke in the acute stages they are often not in a position to understand the risks. Potential benefits, risks and patient opinion need to be clearly stated.

\section{Additional references for Appendix 1}

70. Clave EP, DeKraa M, Arreloa V, et al. The effect of bolus viscosity on swallowing function in neurogenic dysphagia. Aliment Pharm Ther 2006;24:1385-1394.

71. Newman R, Vilardell, N, Clave P, et al. Effect of bolus viscosity on the safety and efficacy of swallowing and the kinematics of the swallow response in patients with oropharyngeal dysphagia: White Paper by the European Society for Swallowing Disorders. Dysphagia 2016;31:232-249.

72. Finestone HM, Foley NC, Woodbury MG, et al. Quantifying fluid intake in dysphagic stroke patients: a preliminary comparison of oral and nonoral strategies. Arch Phys Med Rehab 2001;82:744-746.

73. Steele CM, Alsanei WA, Ayanikalath S, et al. The influence of food texture and liquid consistency modification on swallowing physiology and function: a systematic review. Dysphagia 2015;30:2-6.

74. Cichero JA. Thickening agents used for dysphagia management: effect on bioavailability of water, medication and feelings of satiety. Nutrit J 2013;12:1-8.

75. Vallons KJR, Helmens HJ, Oudhuis AACM. Effect of human saliva on the consistency of thickened drinks for individuals with dysphagia. Int J Lang Comm Dis 2014;50(2):165-75.

76. Beck AM, Kjaersgaard A, Hansen T, et al. Systematic review and evidence based recommendations on texture modified foods and thickened liquids for adults (above 
17 years) with oropharyngeal dysphagia - An updated clinical guideline. Clinical Nutrition 2018;37(6):1980-1991.

77. Kaneoka A, Pisegna JM, Saito H, et al. A systematic review and meta-analysis of pneumonia associated with thin liquid vs. thickened liquid intake in patients who aspirate. Clin Rehab 2017;31(8):1116-1125.

78. Andersen UT, Beck AM, Kjaersgaard A, et al. Systematic review and evidence based recommendations on texture modified foods and thickened fluids for adults (18 years) with oropharyngeal dysphagia. e-SPEN J 2016;8:e127-e134.

79. Foley N, Teasell R, Salter K, et al. Dysphagia treatment post stroke: a systemtic review of randomised controlled trials. Age Ageing 2008;37(3):258-64.

80. Mertz-Garcia J, Chambers E, Molander M. Thickened liquids: practice patterns of speech-language pathologists. Am J Speech-Lang Pat 2005;14:4-13.

81. McCurtin A, Brady R, Coffey K, et al. A tale of clarity and contradictions: speech and language therapist's insights regarding the highly-used treatment of thickened liquids for post-stroke aspiration. Int J Ther Rehab 2019; in press.

82. Colodny N. Dysphagic independent feeders' justifications for noncompliance with recommendations by a speech-language pathologist. Am J Speech-Lang Pat 2005;14:61-70.

83. King JM, Ligman K. Patient noncompliance with swallowing recommendations: reports from speech-language pathologists. Contemp Iss Comm Sci Dis 2011;38:5360.

84. Sharp HM, Bryant KN. Ethical issues in dysphagia: when patients refuse assessment or treatment. Semin Speech Lang 2003;24:285-300. 
85. Logemann JA, Gensler G, Robbins J, et al. A randomized study of three interventions for aspiration of thin liquids in patients with dementia or Parkinson's disease. Speech-Language Hearing Research 2008;51:73-183. 76.

86. McQueen CE, Taubert S, Cotter D, et al. Which commercial thickening agent do patients prefer? Dysphagia 2003;18:46-52.

87. Swan K, Speyer R, Heijnen B, et al. Living with oropharyngeal dysphagia: effects of bolus medication on health related quality of life - a systematic review. Qual Life Res 2015:24:2447-2456.

88. Lim DJH, Mulkerrin SM, Mulkerrin EC, et al. A randomized trial of the effect of different fluid consistencies used in the management of dysphagia on quality of life: a time trade-off study. Age Ageing 2016;45:309-312.

89. Sura L, Madhavan A, Carnaby G, et al. Dysphagia in the elderly: management and nutritional considerations. Clin Int Aging 2012;7:287-298.

90. O'Keefe ST. Use of modified diets to prevent aspiration in oropharyngeal dysphgia: is current practice justified? BMC Geriatrics 2018;18:167. Available at: O’Keeffe https://doi.org/10.1186/s12877-018-0839-7. Accessed December 132018.

91. Pownall S, Taylor C. Use of thickening agents and nutritional supplements for patients with dysphagia following stroke. Brit J Neurosci Nurs 2017;13:260-8.

92. Murray J, Doeltgen S, Miller M, et al. A survey of thickened fluid prescribing and monitoring practices of Australian health professionals. J Eval Clin Pract 2014;20:596-600.

93. Glassburn DL, Deem JF. Thickener Viscosity in Dysphagia Management: Variability among Speech-Language Pathologists. Dysphagia 1998;13:218-222.

94. Murray J, Miller M, Doeltgen S, et al. Intake of thickened liquids by hospitalized adults with dysphagia after stroke. Int J Speech-Lang Pat 2014:16(5):486-494. 
TEKA

95. Fraser S, Steele, CM. The Effect of the Chin-Down Position on Penetration Aspiration in adults with Dysphagia. Can J Speech-Lang Pat 2012:36(2):142-148.

96. Saconato M, Chiari MB, Lederman HM, et al. Effectiveness of the Chin Tuck Manoeuvre to Facilitate Swallowing in Neurologic dysphagia. Int Arch Otorhinolaryngol 2016;20(1):013-017

97. Sommerville, P, Lang A, Nightingale S, et al. Dysphagia after stroke and feeding with acknowledged risk. Brit J Neurosci Nurs 2016;12(4):162-169. 
TEKA

Tables

Table 1 TEKA components for the intervention evaluation

\begin{tabular}{|c|c|}
\hline Knowledge form & Examples /definitions \\
\hline $\begin{array}{l}\text { description } \\
\text { Information which } \\
\text { describes and } \\
\text { explains the } \\
\text { treatment in } \\
\text { question }\end{array}$ & $\begin{array}{l}\text { Classification - Category of intervention into which the treatment fits. } \\
\text { Definition - Statement which describes the treatment. } \\
\text { Procedures - Steps demonstrating exactly how to carry out treatment. } \\
\text { Theory - The proposal or hypothesis that describes the actual nature of the } \\
\text { process that transforms the intervention into the desired outcome. } \\
\text { Aims - The specific goals of treatment - skills / functions targeted and to what } \\
\text { intent. } \\
\text { Ingredients - What the clinician does/actions taken in order to effect change } \\
\text { in the targeted areas. } \\
\text { Mechanism of action - The specific mechanism by which an effect/change is } \\
\text { produced. } \\
\text { Candidacy criteria - Criteria (inclusion/exclusion) by which patients are } \\
\text { pseudoscientific features (e.g., being authority based, immune to testing, } \\
\text { having an implausible theory). } \\
\text { considered appropriate for the treatment and why. } \\
\text { Evaluation criteria - Outcome measures/tools and behaviours which provide } \\
\text { basis for evaluating treatment success. } \\
\text { Red flags - Whether the treatment raises concerns such as having } \\
\text { Rion - Knowledge regarding reported / known risks / side effects } \\
\text { ing the treatment. }\end{array}$ \\
\hline
\end{tabular}




\begin{tabular}{|c|c|}
\hline $\begin{array}{l}\text { Knowledge about } \\
\text { other treatments } \\
\text { which target the } \\
\text { same areas of need }\end{array}$ & $\begin{array}{l}\text { Other treatments - Identification / contemplation of other potential } \\
\text { treatments which target the same problem, the comparison of these with the } \\
\text { treatment under consideration and in the context of the individual in question } \\
\text { and the context in which the treatment is to be provided. The "no treatment" } \\
\text { option should also be considered, taking into account potential benefits, risks } \\
\text { and patient preferences. }\end{array}$ \\
\hline $\begin{array}{l}\text { Research evidence } \\
\text { Results or } \\
\text { outcomes of } \\
\text { efficacy research }\end{array}$ & $\begin{array}{l}\text { Systematic reviews - Summaries including systematic reviews and meta- } \\
\text { analyses of the results of high-level, well-designed intervention studies. } \\
\text { Randomised control trials - Experiments where bias is reduced as } \\
\text { participants are allocated at random either to receive an intervention or to a } \\
\text { control group } \\
\text { Observational studies - Studies in which participants are observed in a } \\
\text { systematic manner/certain outcomes are measured, with no attempt made to } \\
\text { affect the outcome. } \\
\text { Case series - A group or series of case studies involving patients who are } \\
\text { given a similar treatment with no comparison group, but where participants } \\
\text { may act as their own controls. } \\
\text { Single case studies - Reports on single cases which involve forms of control } \\
\text { i.e. treated / untreated items, repeated measures at baseline and the client } \\
\text { the evidence /make recommendations regarding specific interventions - where } \\
\text { acting as his/her own control. }\end{array}$ \\
\hline Practice evidence & $\begin{array}{l}\text { Individual clinical opinion - The experience / opinion of an individual } \\
\text { clinician (e.g., the person providing the treatment in the immediate clinician- }\end{array}$ \\
\hline
\end{tabular}




\begin{tabular}{|c|c|}
\hline $\begin{array}{l}\text { accruing } \\
\text { from clinical }\end{array}$ & $\begin{array}{l}\text { patient dyad) or the opinion of other individual clinicians - for example, } \\
\text { retrieved via blogs. } \\
\text { Collective clinical voice - Peer-reviewed studies which include results (e.g., } \\
\text { perceptions, experiences, attitudes) from a representative sample of healthcare } \\
\text { providers who have experience of providing the treatment in question. } \\
\text { Clinical consensus - The combined experience /consensus of a number of } \\
\text { clinicians providing the treatment either locally, nationally or internationally } \\
\text { based. } \\
\text { Expert opinion - The experience/opinion of individuals or groups of people } \\
\text { who are specialists / experts in the field / intervention. } \\
\text { Clinical practice guidelines - Guidelines which make recommendations } \\
\text { regarding specific interventions - where that recommendation is based on the } \\
\text { consensus, usually of the guideline development group, who may or may not } \\
\text { have experience of the specified intervention. } \\
\text { organisations / working groups regarding that same intervention. }\end{array}$ \\
\hline $\begin{array}{l}\text { Patient evidence } \\
\text { Knowledge } \\
\text { accruing } \\
\text { from patient }\end{array}$ & $\begin{array}{l}\text { Individual patient opinion (intervention recipient) - The opinion of the } \\
\text { person using/potentially using the treatment regarding that treatment. } \\
\text { Other patients' experience of intervention - the opinion of singular patients } \\
\text { who have previously been in receipt of the treatment. } \\
\text { Carergiver opinion - The opinion of the carer (partner, spouse etc.) regarding } \\
\text { the treatment. } \\
\text { Patient values \& preferences - The values/preferences of the person and (or } \\
\text { their carers) (potentially) using the treatment. }\end{array}$ \\
\hline
\end{tabular}




\begin{tabular}{|c|c|}
\hline & $\begin{array}{l}\text { Collective patient voice - Peer-reviewed studies which include results (e.g. } \\
\text { perceptions, experiences, attitudes) from a representative sample of healthcare } \\
\text { users, usually with the same presenting condition and who have experience of } \\
\text { utilising the treatment in question. } \\
\text { Individual clinical evidence - Evidence from clinical assessment / } \\
\text { intervention with the individual in question, which is relevant to the treatment } \\
\text { decision-making because of demonstrated response to the treatment. } \\
\text { Patient groups - Experience of patient groups not directly involved in } \\
\text { treatment delivery. Usually obtained via patient websites or advocacy groups. }\end{array}$ \\
\hline $\begin{array}{l}\text { Contextual } \\
\text { evidence } \\
\text { Knowledge, } \\
\text { typically practical } \\
\text { in nature, accruing } \\
\text { from and } \\
\text { regarding factors } \\
\text { which } \\
\text { impact treatment } \\
\text { implementation }\end{array}$ & $\begin{array}{l}\text { Cost - The direct cost of providing the treatment and the resources available } \\
\text { from both the healthcare provider and healthcare user. } \\
\text { Healthcare policy - Local / national/insurer policies related to the treatment / } \\
\text { provision of services. } \\
\text { Resources - Resources required to support treatment implementation, } \\
\text { including but not limited to: Access to equipment needed to implement the } \\
\text { treatment; staffing requirements; the training required (e.g. type, intensity, } \\
\text { length and cost) and the ability of the service to provide training; and the } \\
\text { potential costs attached to equipment monitoring / maintenance / repair over a } \\
\text { sustained period. } \\
\text { Feasibility of protocol - The ability to provide / implement the treatment in } \\
\text { its original form, which may impact treatment adherence/fidelity. }\end{array}$ \\
\hline $\begin{array}{l}\text { Judico-ethics } \\
\text { based evidence }\end{array}$ & $\begin{array}{l}\text { The law of the land - The laws of the country / state where the treatment is } \\
\text { being provided. } \\
\text { Legal principles - Tenets guiding good practice, such as duty of care, breach } \\
\text { of duty, reasonableness, do no harm. }\end{array}$ \\
\hline
\end{tabular}


issues and

regarding the

treatment which

reflects on moral

and ethical values
Basic ethical principles - Principles such as informed consent for treatment and capacity to give consent.

Impact issues- Issues pertaining to patient impact such as the influence of the treatment on quality of life, consideration of treatment benefit vs. treatment burden and expense impact (economic/time) on the patient.

Commercial drivers - Drivers outside the immediate decision-making arena, such as whether the treatment is commercially driven / has vested interests, and how this might impact decision-making / available information. 
Table 2 Sample questions for intervention description exercise

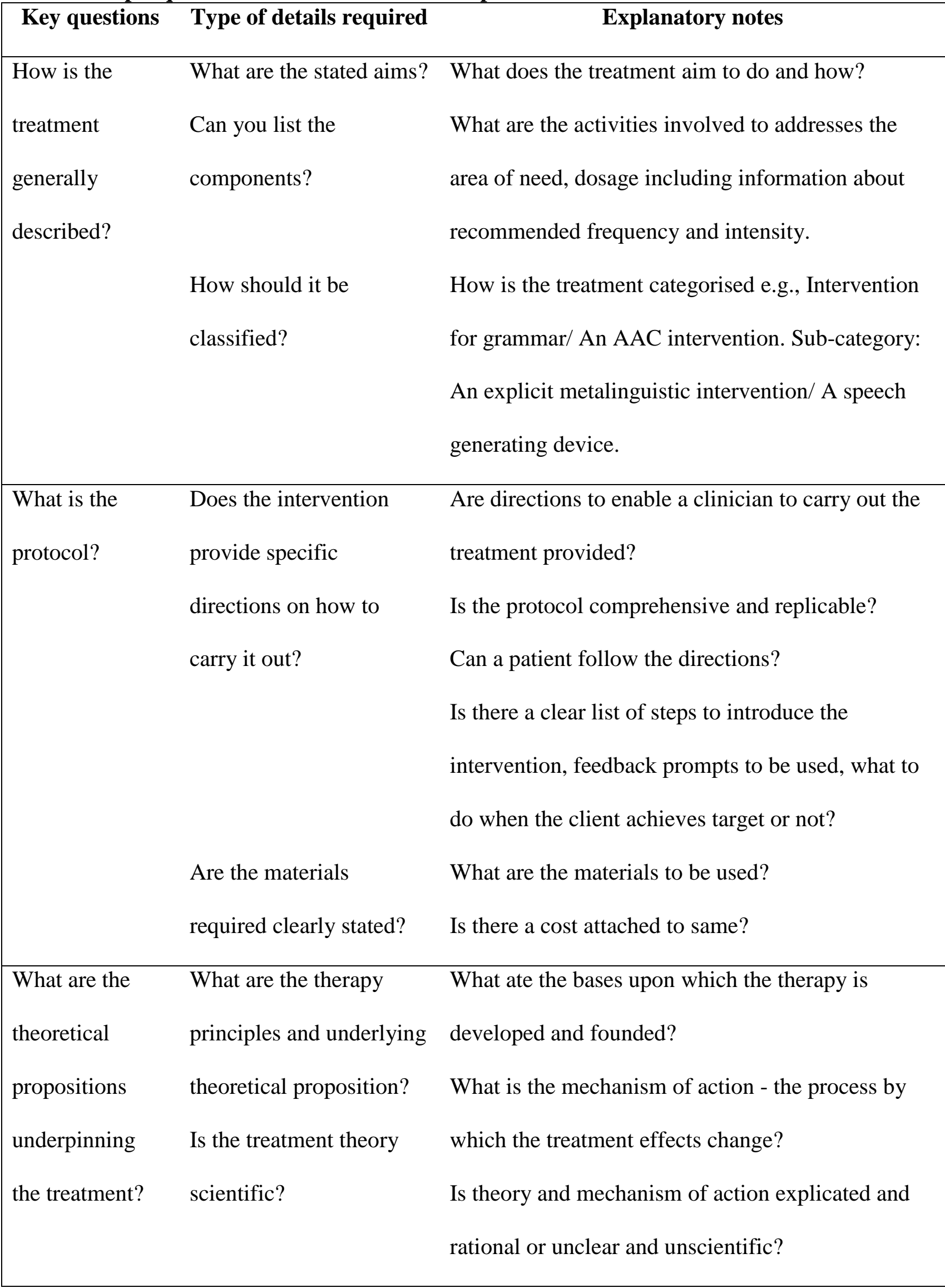




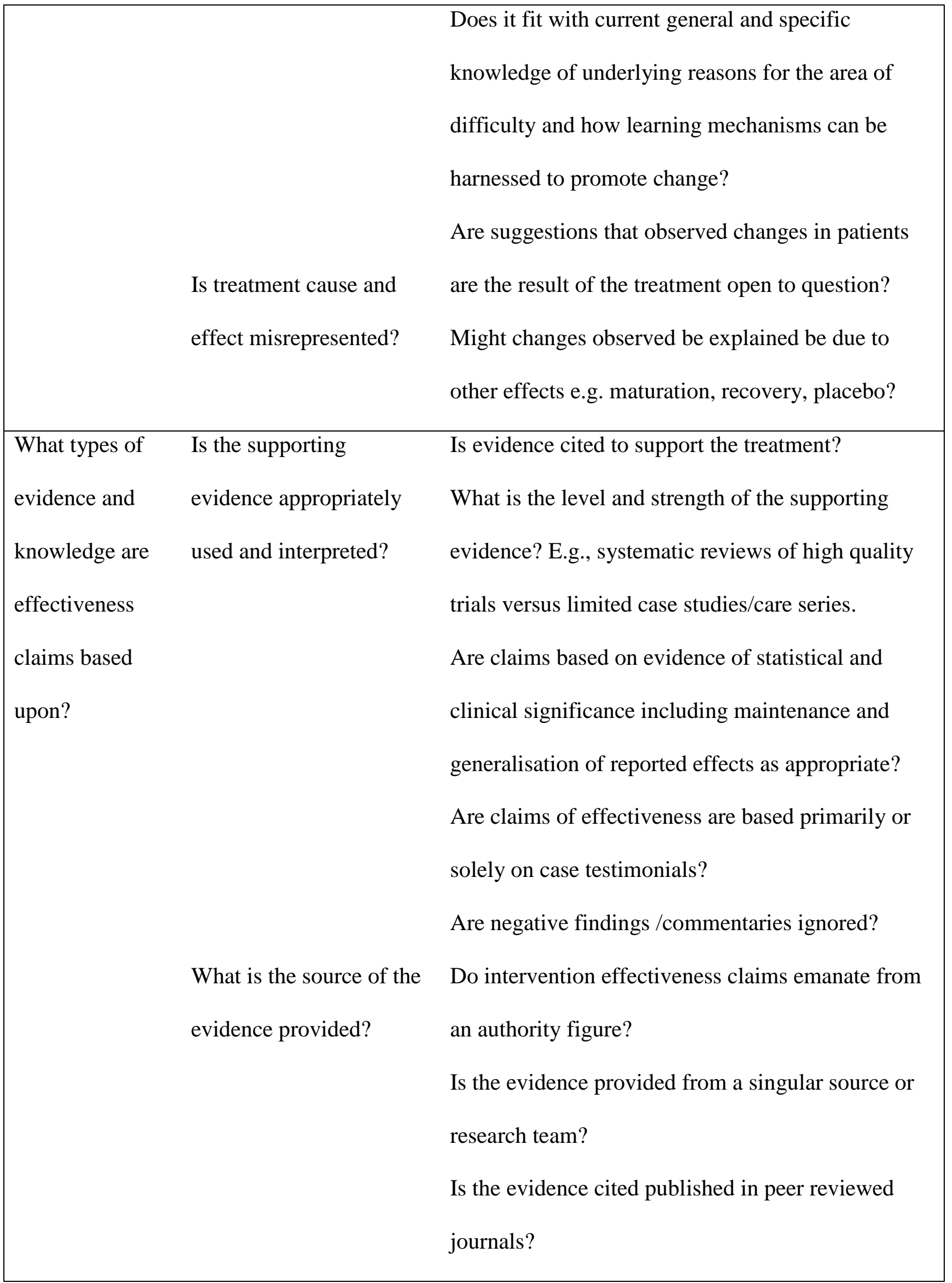




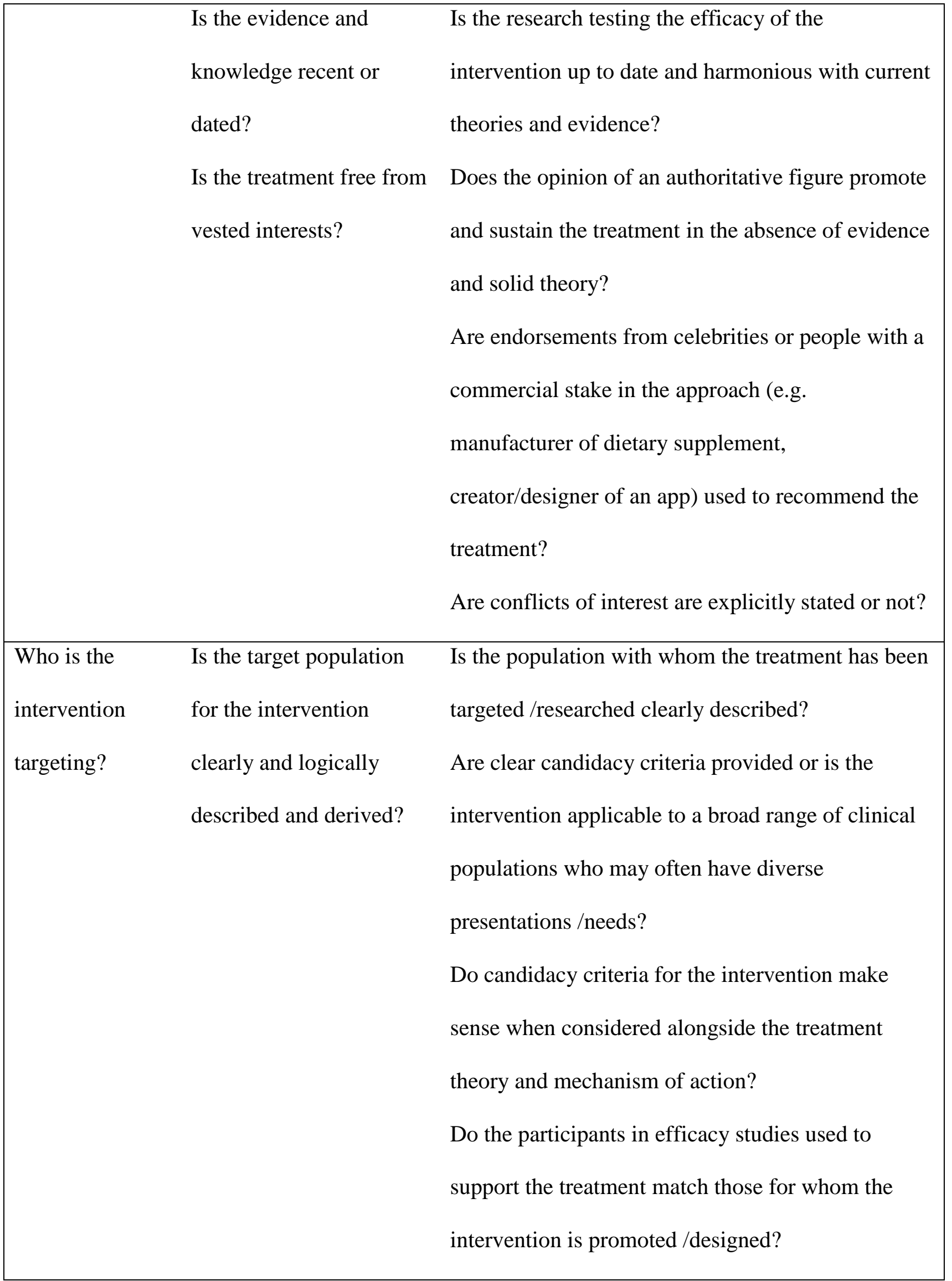




\begin{tabular}{|c|c|c|}
\hline & & $\begin{array}{l}\text { Does the nature of the treatment fit with the } \\
\text { developmental stage and/or nature of difficulty } \\
\text { and/or aetiology/underlying deficits and/or client } \\
\text { strengths and needs? }\end{array}$ \\
\hline $\begin{array}{l}\text { What outcomes } \\
\text { are used? }\end{array}$ & $\begin{array}{l}\text { Are outcomes provided } \\
\text { and how can they be } \\
\text { described? }\end{array}$ & $\begin{array}{l}\text { Are outcomes measureable? } \\
\text { Do outcomes measure what was targeted in the } \\
\text { intervention? } \\
\text { Are outcome measures provided? } \\
\text { What types of outcomes are employed? } \\
\text { Quantitative tools? Judgment based measures? } \\
\text { Criterion referenced measures? } \\
\text { Are the tools employed validated? Reliable? Have } \\
\text { they good intra and inter-rater reliability? }\end{array}$ \\
\hline & $\begin{array}{l}\text { Can outcomes be } \\
\text { attributed solely to the } \\
\text { intervention in question? }\end{array}$ & $\begin{array}{l}\text { Have non-specific intervention variables such as the } \\
\text { therapeutic alliance or a placebo effect been } \\
\text { considered in evaluating outcomes? } \\
\text { Can service delivery effects such as treatment } \\
\text { intensity explain outcomes? } \\
\text { Do measures account for /distinguish between } \\
\text { proximal and distal outcomes e.g., proximal = a } \\
\text { change in the area targeted such as swallow } \\
\text { efficacy; distal = increased confidence swallowing. }\end{array}$ \\
\hline & $\begin{array}{l}\text { Are patient reported } \\
\text { outcome measures } \\
\text { (PROMs) used? }\end{array}$ & $\begin{array}{l}\text { Are PROMs employed to measure patient report } \\
\text { changes? Are PROMs the sole outcome measure } \\
\text { employed? }\end{array}$ \\
\hline
\end{tabular}




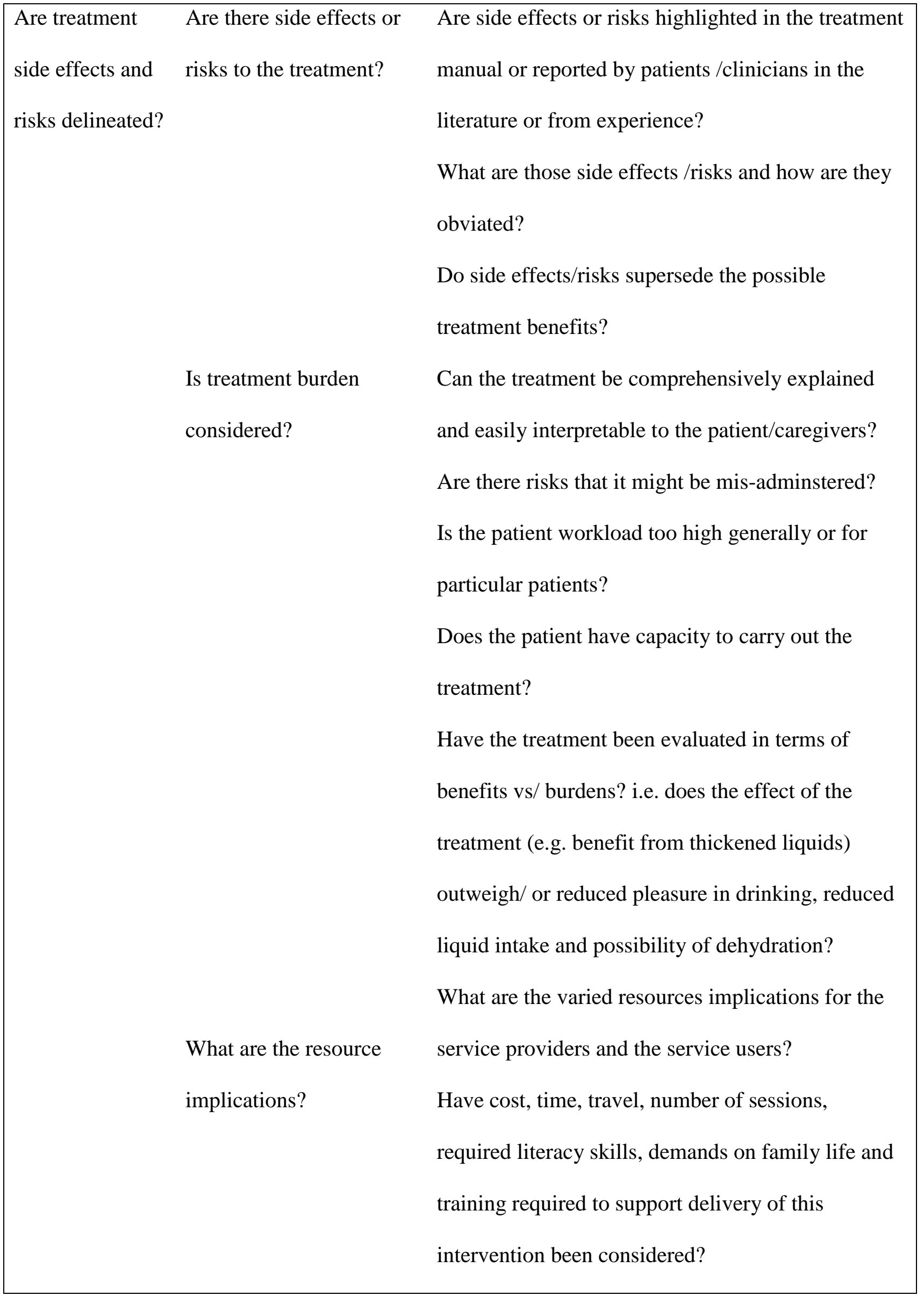


TEKA

Are the resource requirements rationalized in terms

of the treatment benefits? 
TEKA

Table 3 Summary points of intervention evaluation exercise for illustrative example

\begin{tabular}{|c|c|}
\hline form & Summary points of thickened liquids (TL) evaluation \\
\hline $\begin{array}{l}\text { Intervention } \\
\text { description }\end{array}$ & $\begin{array}{l}\text { General: } \\
\text { 1. TL treats aspiration. } \\
\text { 2. Drinks can be prepared in various consistencies so the treatment can be } \\
\text { individualised. } \\
\text { 3. TL products are gum or starch based. } \\
\text { 4. TL is purported to slow the bolus, which potentially enables improved swallow } \\
\text { safety. } \\
\text { 5. Outcomes are typically demonstrated via instrumentation (especially for silent } \\
\text { aspirators), a reduction in coughing on drinking and absence of/ reduction in } \\
\text { occurrence of aspiration/aspiration pneumonia. } \\
\text { 6. Improvements may result from other factors in this population (e.g. spontaneous } \\
\text { recovery). }\end{array}$ \\
\hline & $\begin{array}{l}\text { Side effects/risks: } \\
\text { 1. Effects on oral/pharyngeal transit times. } \\
\text { 2. Increases oral/pharyngeal residue. } \\
\text { 3. Increased risk of dehydration. } \\
\text { 4. Poor at quenching thirst. } \\
\text { 5. Poor flavour release. } \\
\text { 6. Effects of delayed dissolution of some medications. } \\
\text { 7. Starch-based thickeners retain consistency with human saliva less well. } \\
\text { 8. Oral effort may be increased. }\end{array}$ \\
\hline
\end{tabular}




\begin{tabular}{|c|c|}
\hline $\begin{array}{l}\text { Alternative } \\
\text { treatment }\end{array}$ & $\begin{array}{l}\text { 1. Free water protocols have limited evidence but are also under-researched. } \\
\text { 2. Free water protocols require good oral hygiene to be maintained. } \\
\text { 3. Risk feeding is a possibility. } \\
\text { 4. Neither alternative is currently employed in the organisation. } \\
\text { 5. Risk protocols are currently being developed. } \\
\text { 6. Postural manoeuvres are an option. They have limited evidence and would need to } \\
\text { be evaluated and monitored. } \\
\text { 7. In the early stages post-stroke, patients may not be able to carry out "complex" } \\
\text { treatments. }\end{array}$ \\
\hline $\begin{array}{l}\text { Research } \\
\text { evidence }\end{array}$ & $\begin{array}{l}\text { 1. Consensus from some recent systematic reviews is that there is some evidence for } \\
\text { TL reducing the risk of airway invasion. } \\
\text { 2. Most reviews are not specific to the stroke population. } \\
\text { 3. Reviews conclude that the evidence in favour of TL is not strong. } \\
\text { 4. Most stroke clinical guidelines recommend TL either in isolation or as part of } \\
\text { multi-component programme. } \\
\text { 5. Stroke clinical guideline recommendations for TL tend not be evidence based. }\end{array}$ \\
\hline $\begin{array}{l}\text { Practice } \\
\text { evidence }\end{array}$ & $\begin{array}{l}\text { 1. Most speech-language pathologists (SLPs) working with people with dysphagia } \\
\text { post-stroke use the thickened liquids intervention. } \\
\text { 2. Intervention use is pivoted on safety-first reasoning. } \\
\text { 3. The intervention is used as a first step on the treatment ladder. } \\
\text { 4. SLPs believe thickening drinks is an effective intervention. } \\
\text { 5. SLPs perceive there to be limited treatment options for aspiration. }\end{array}$ \\
\hline
\end{tabular}




\begin{tabular}{|c|c|}
\hline & $\begin{array}{l}\text { 6. SLPs believe multiple local, individual, and contextual factors impact on treatment } \\
\text { effectiveness. }\end{array}$ \\
\hline & $\begin{array}{l}\text { Local } \\
\text { 1. I (the clinician) have } 10 \text { years clinical experience working in acute settings with } \\
\text { people with dysphagia including post-stroke. } \\
\text { 2. I have used thickened liquids frequently to treat aspiration risk. } \\
\text { 3. I find it generally effective, especially in cases where patients need time to initiate } \\
\text { a swallow. } \\
\text { 4. My perceptions of effectiveness are based primarily on reduced clinical signs, and } \\
\text { I repeat instrumental evaluations where available. } \\
\text { 5. Many stroke patients are discharged before I can follow them up. } \\
\text { 6. My colleagues agree it is a useful treatment in the acute stages. } \\
\text { 7. I have discussed this intervention with an expert who advises that individual } \\
\text { patient clinical evidence and patient opinion is the best way to evaluate effectiveness } \\
\text { and usabilty. }\end{array}$ \\
\hline $\begin{array}{l}\text { Patient } \\
\text { evidence }\end{array}$ & $\begin{array}{l}\text { General } \\
\text { 1. People with dysphagia across a range of conditions tend to dislike thickened } \\
\text { liquids. } \\
\text { 2. Non-adherence rates vary between } 40 \% \text { and } 80 \% \text {. } \\
\text { 3. People with dysphagia prefer non- thickened liquids treatments. } \\
\text { 4. People with dysphagia post-stroke dislike this intervention, which may impact on } \\
\text { adherence and hydration. } \\
\text { 5. Lack of sensory appeal is important in framing dislike of this intervention. } \\
\text { 6. Involvement in and understanding of reasons for thickening can be poor in people }\end{array}$ \\
\hline
\end{tabular}




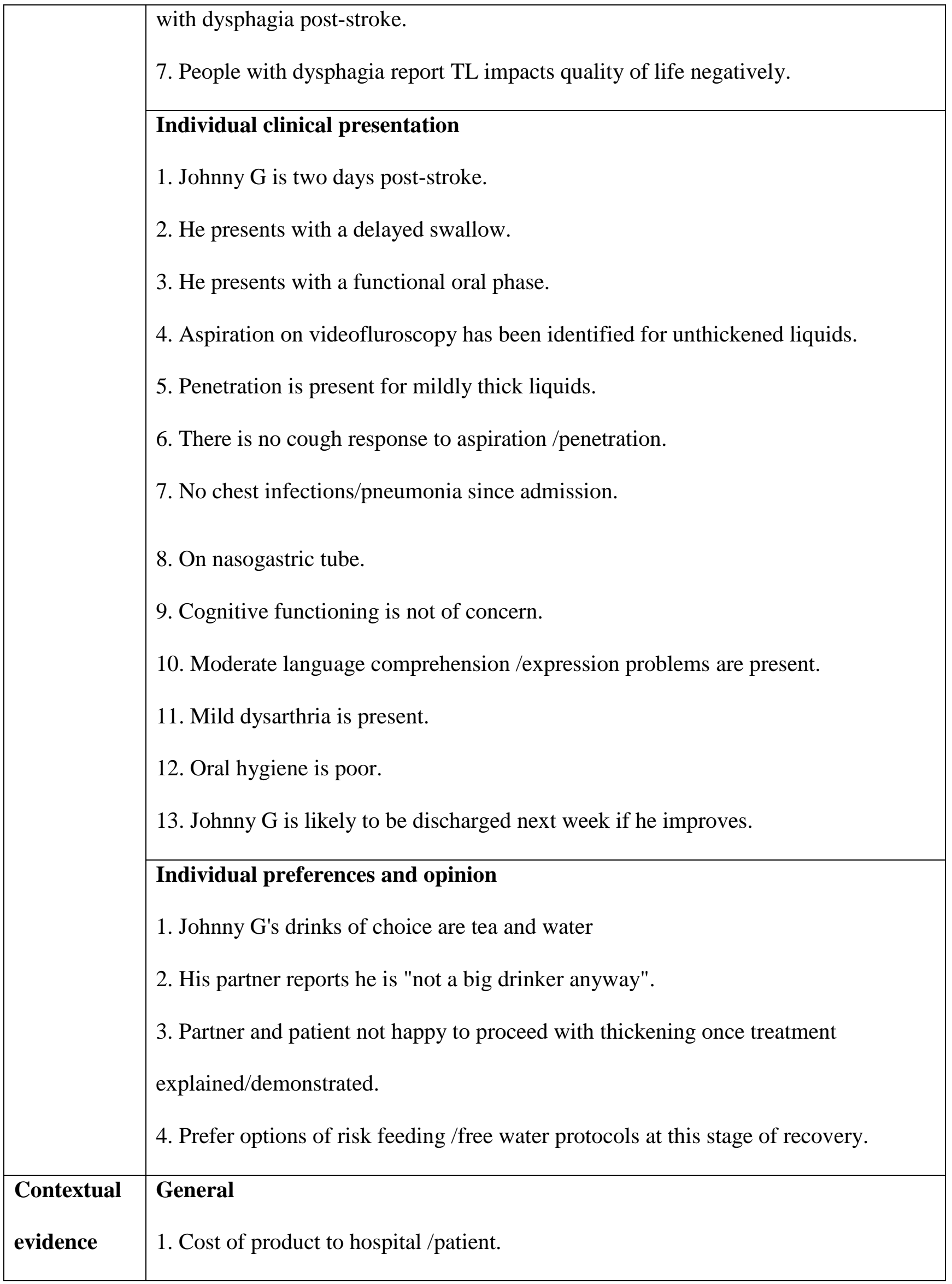




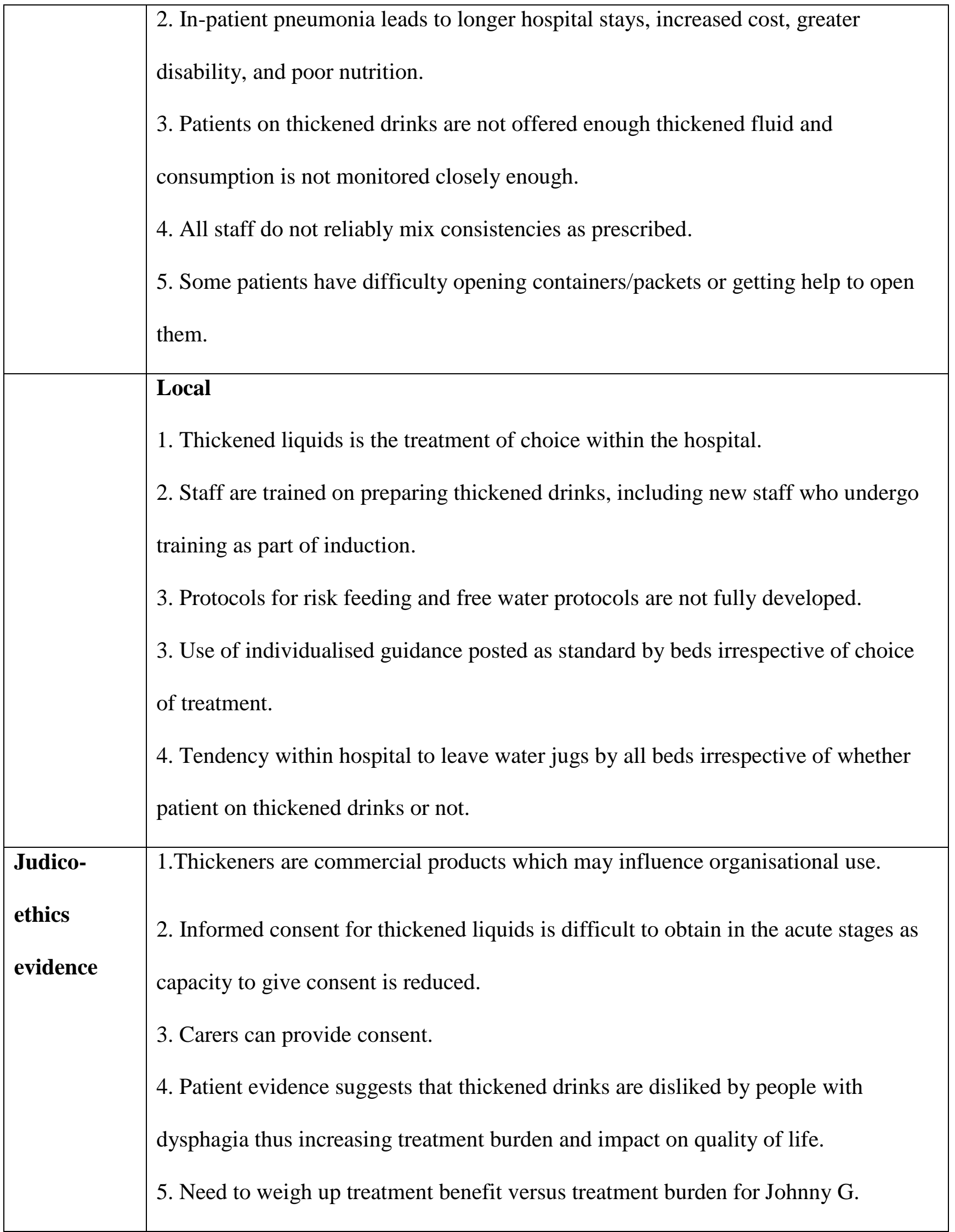


TEKA 